\title{
EVALUACIÓN DE DOS TÉCNICAS DE ACODAMIENTO PARA LA PROPAGACIÓN VEGETATIVA DEL ALGARROBO Prosopis chilensis (Mol) Stuntz
}

\author{
TARNOWSKI, C. G. ${ }^{1 *}$
}

\begin{abstract}
RESUMEN
El objetivo general del ensayo fue evaluar el uso de dos métodos de propagación vegetativa en P. chilensis: (A) acodo aéreo y (B) en montículo. En (A) se emplearon árboles de 3,5 años de edad y se ensayaron en dos épocas (primavera-verano), con AIB $1 \%$ y el control testigo. En (B) se usaron plantas de 8 meses de edad, anillado con alambre y diferentes tipos de sustratos: arena, turba y una mezcla de ambos. Se obtuvieron 42,5\% de acodos enraizados en primavera con AIB y un promedio de 14,5 raíces por acodo aéreo, sin diferencias con respecto al verano. En (B), el máximo porcentaje de enraizamiento fue del 75 \% en el sustrato arena, con alambre y un promedio de 3,89 raíces por acodo. En (A) se concluye que AIB mejora el porcentaje de enraizamiento en ambas épocas; y en (B) el alambre favorece el enraizamiento de los acodos en montículo en presencia del sustrato arena.
\end{abstract}

Palabras clave: auxina AIB, sustratos, diferentes estaciones.

\begin{abstract}
Evaluation of two layering techniques for the vegetative propagation of algarrobo Prosopis chilensis (Mol) Stuntz.

The aim of this work was to evaluate two methods of vegetative propagation in $P$. chilensis: air layering (technique A) and by mound (B). For (A) trees of 3.5 years old were employed. The trial consisted in two treatments (IBA $1 \%$ and control without hormone) applied in two seasons (spring-summer). For (B) 8-month-old plants were employed. The trial consisted in two treatments (plants wire ringed and without ring) and 3 different substrates as mound: sand, peat and a mixture
\end{abstract}

1.- Estación Experimental Agropecuaria La Consulta, Centro Regional Mendoza-San Juan. CC 8. (5567) La Consulta, Mendoza, Argentina. Email: tarnowski.christian@inta.gob.ar Manuscrito recibido el 30 de marzo de 2020 y aceptado para su publicación el 7 de agosto de 2020.

Tarnowski, CG. Evaluación de dos técnicas de acodamiento para la propagación vegetativa del algarrobo Prosopis chilensis (Mol) Stuntz. FAVE - Ciencias Agrarias 20 (1): 305-315. CC BY-NC-SA 4.0 


\section{G. Tarnowski}

of both. In technique A $42.5 \%$ of rooted aerial layers were obtained in spring with AIB and an average of 14.5 roots per layer, with no differences compared to summer. In technique $B$ the maximum rooting percentage was $75 \%$ in the sand substrate with an average of 3.89 roots per layer. In $A$, it is concluded that IBA improved the rooting percentage in both seasons. In B, the wire ringing favored the rooting of the layers in mound with sand as substrate.

Key words: IBA hormone, substrates, layering season.

\section{INTRODUCCIÓN}

En Argentina se denomina con el nombre común de algarrobos a varias especies del género Prosopis y seis de ellas son las más importantes $(P$. chilensis, $P$. flexuosa DC., P. alba Grisebach, P. nigra (Grisebach) Hieronymus, $P$. hassleri Harms y $P$. caldenia Burkart) (Burkart 1952, Palacios et al. 1988). Estos árboles son uno de los principales recursos naturales explotados por el hombre por ser consideradas especies multipropósitos: proveen madera durable para mueblería fina y para la carpintería rural; un elevado poder calórico de la leña y el carbón; frutos con alto contenido de azúcares y proteínas aptos para el consumo humano y de excelente calidad forrajera; el néctar de sus flores favorece la producción de miel; se lo emplea también para la protección del suelo contra la erosión y la desertificación de ambientes degradados en zonas áridas y semiáridas; constituyen un elemento de enorme importancia actual y potencial en los sistemas silvopastoriles y agroforestales en amplias áreas de la región chaqueña y la capacidad de producir sombra en ambientes semidesérticos (Verga et al. 2005).

La multiplicación del algarrobo a través de semillas es la forma más común y económica de obtención de plantines (Tapia et al. 2005), pero si el interés es obtener una descendencia que sea homogénea, esta forma de propagación no sería la más adecuada debido a que existe una elevada variabilidad genética intra e interespecífica provocado en algunos casos por los procesos de hibridación natural que se producen entre las especies de Prosopis (Verga et al. 2005). Esto implica que el material genético que se utilice tendrá una alta variabilidad, aun procediendo de la misma planta madre.

Para ello, se recurren a los métodos de propagación asexual los cuales permiten producir una población más homogénea de aquellos individuos que fueron elegidos por sus características deseables, (ej. ausencia de espinas) ya sea para la instalación de rodales semilleros clonales o para obtener una plantación comercial uniforme (Carpineti 2005).

El acodamiento es un método de propagación vegetativa por el cual se induce la formación de raíces adventicias en la rama de un árbol cuando aún permanece unida a la planta madre. El brote enraizado, es entonces cortado de la planta madre para crecer sobre sus propias raíces, originando una nueva planta. Es un método alternativo para algunas especies de difícil enraizamiento por estacas, pues además de evitar el estrés hídrico causado por la ausencia de raíces, dispone de nutrimentos durante todo 
el período de enraizamiento. Hay tres tipos de acodamientos: aéreo, en montículo y en trinchera (Hartmann y Kester 1987).

Existen algunas experiencias en relación a la propagación del género Prosopis mediante el acodo aéreo. En México, Ramírez-Malagón et al. (2014) usaron esta técnica para clonar P. laevigata (Humb. \& Bonpl. ex Willd.) M.C. Johnst y obtuvieron un $90 \%$ de acodos enraizados; en Nigeria, Abdou et al (2015) realizaron 60 acodos en 15 árboles de P. africana (Guill. et Perr.) Taub. y registraron en promedio $28 \%$ de acodos enraizados. Hagen y Palzkill (1989) lograron enraizar hasta un $100 \%$ de acodos en tres árboles de P. chilensis. En el país, un grupo de investigadores de la Facultad de Recursos Naturales de la Universidad Nacional de Formosa realizaron varios ensayos de acodos aéreos en P. alba para evaluar la influencia del factor época del año (Vega et al. 1999); el efecto de diferentes auxinas (Vega et al. 2002b) y la interacción del genotipo (Vega et al. 2002a).

El objetivo general del presente trabajo fue evaluar el uso de dos métodos de propagación vegetativa basado en técnicas de acodamiento: (1) acodo aéreo, cuyos objetivos específicos fueron a) determinar el efecto de la hormona enraizante ácido indolbutírico (AIB) y b) evaluar la incidencia de la época de realización; (2) acodo en montículo, con los objetivos de a) determinar el efecto del estrangulado de los brotes y b) evaluar la influencia del tipo de sustrato.

\section{MATERIALES Y MÉTODOS}

Los ensayos fueron realizados en la Estación Experimental de Cultivos Tropicales Yuto del INTA en la provincia de Jujuy, Argentina, la cual se encuentra ubicada en el Pedemonte de la Selva de Yungas y presenta las siguientes características climáticas: temperatura media anual de $20,7^{\circ} \mathrm{C}$ y precipitaciones de $862 \mathrm{~mm}$ (Del Castillo y Tarnowski 2006).

\section{Acodo aéreo}

Determinación del efecto de la hormona enraizante ácido indolbutírico (AIB) y evaluación de la incidencia de la época de realización. Para este ensayo se utilizaron plantas de $P$. chilensis provenientes de árboles semilleros identificados en parcelas experimentales del INTA Yuto. Dichas plantas tenían 3,5 años de edad y en promedio una altura de $2,5 \mathrm{~m}$. Se usaron ramas semileñosas que crecieron en el año y tenían entre 0,5 y $1 \mathrm{~cm}$ de diámetro y mínimo 30 $\mathrm{cm}$ de longitud, que fueron seleccionadas aleatoriamente de la parte media y basal de diferentes árboles. En cada rama se realizó un anillado que consistió en un descortezado de $10 \mathrm{~mm}$ de ancho alrededor del tallo. Sobre la lesión practicada se aplicó con un pincel AIB mezclado con talco inerte en dos concentraciones diferentes: $1 \%$ y control sin hormona. El sustrato utilizado fue turba rubia humedecida que se acomodó de tal manera de cubrir alrededor de toda la región tratada. Seguidamente se envolvió con una bolsita plástica de polietileno transparente y una vez por semana se procedió a controlar el estado de humedad del sustrato (Figura 1). 
C. G. Tarnowski

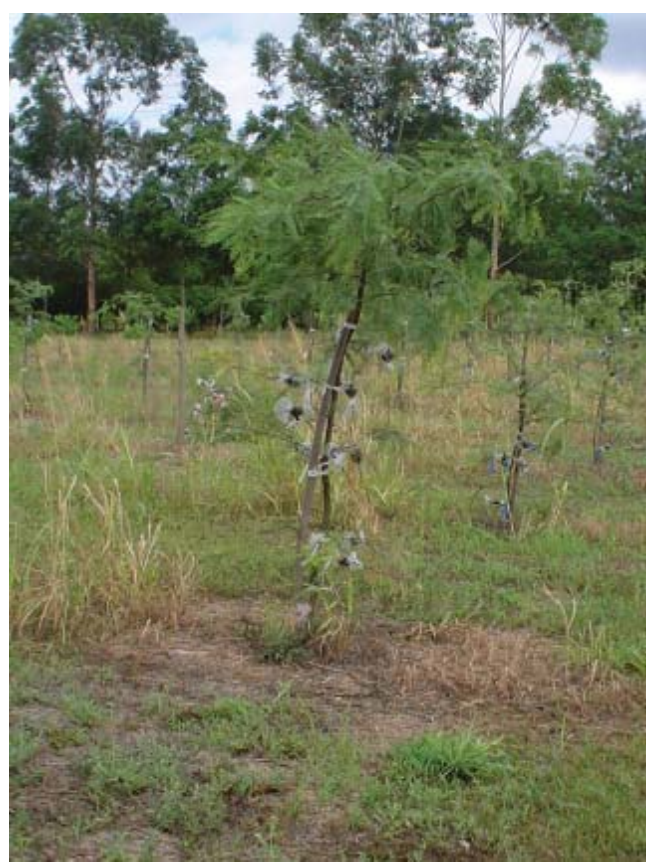

Figura 1. Árboles de Prosopis chilensis de 3 años de edad con acodos aéreos Figure 1. Air layering on Prosopis chilensis 3-year-old trees

Estas tareas fueron hechas en dos momentos del crecimiento de la planta: al inicio de la primavera (fin de septiembre) y luego a inicio del verano (mediados de diciembre) del mismo año. En cada época se realizaron cuatro repeticiones con 10 acodos por unidad experimental en un diseño completamente aleatorizado, los cuales se detallan en la Tabla 1.

\section{Acodo en montículo}

Determinación del efecto del estrangulado de los brotes y evaluación de la influencia del tipo de sustrato. Para este ensayo se utilizaron plantas de $P$. chilensis producidas a partir de semillas provenientes de árboles se- milleros ubicados en parcelas experimentales del INTA Yuto. Las plantas tenían 8 meses de edad, fueron criadas en macetas de $10 \mathrm{~L}$ de capacidad con sustrato tierra de monte y acondicionadas en el vivero bajo una media sombra. El tratamiento de anillado consistió en colocar un alambre de $1 \mathrm{~mm}$ de diámetro alrededor del tallo, a una altura de $15 \mathrm{~cm}$ desde la base de la planta. El control testigo fueron las plantas sin anillar. Seguidamente, alrededor de cada tallo tratado se colocaron tubetes de $250 \mathrm{~cm}^{3}$ cortados longitudinalmente, los cuales fueron sujetados mediante estacas clavadas en la maceta y luego fueron rellenados con tres tipos diferentes de sustrato: arena fina (A), turba parda comercial marca "Kekkilä" (T) y una mezcla de ambos (AT) en una proporción 1:3 (v/v) (Figura 2). 
Para cada uno de estos tratamientos se realizaron tres repeticiones de ocho acodos cada uno, dando un total de 24 plantas por tratamiento, bajo un diseño en Bloques Completos al Azar. En la Tabla 2 se indican los tipos de tratamientos y el número de acodos realizados en cada uno de ellos.

\section{Análisis estadístico}

La evaluación de ambos ensayos se efectuó a los 60 días. Las variables consideradas para evaluar el efecto de los tratamientos fueron el porcentaje de enraizamiento y el número de raíces primarias. Para ello

Tabla 1. Tratamientos de acodo aéreo realizados en diferentes épocas, con y sin aplicación de AIB Table 1. Air layering treatments carried out at different seasons, with and without application of AIB

\begin{tabular}{|c|c|c|}
\hline $\begin{array}{c}\text { Época de } \\
\text { realización }\end{array}$ & AIB & $\begin{array}{c}\mathrm{N}^{\circ} \text { de } \\
\text { acodos }\end{array}$ \\
\hline \multirow{2}{*}{ Primavera } & $1 \%$ & 40 \\
\cline { 2 - 3 } & $0 \%$ & 40 \\
\hline \multirow{2}{*}{ Verano } & $1 \%$ & 40 \\
\cline { 2 - 3 } & $0 \%$ & 40 \\
\hline
\end{tabular}

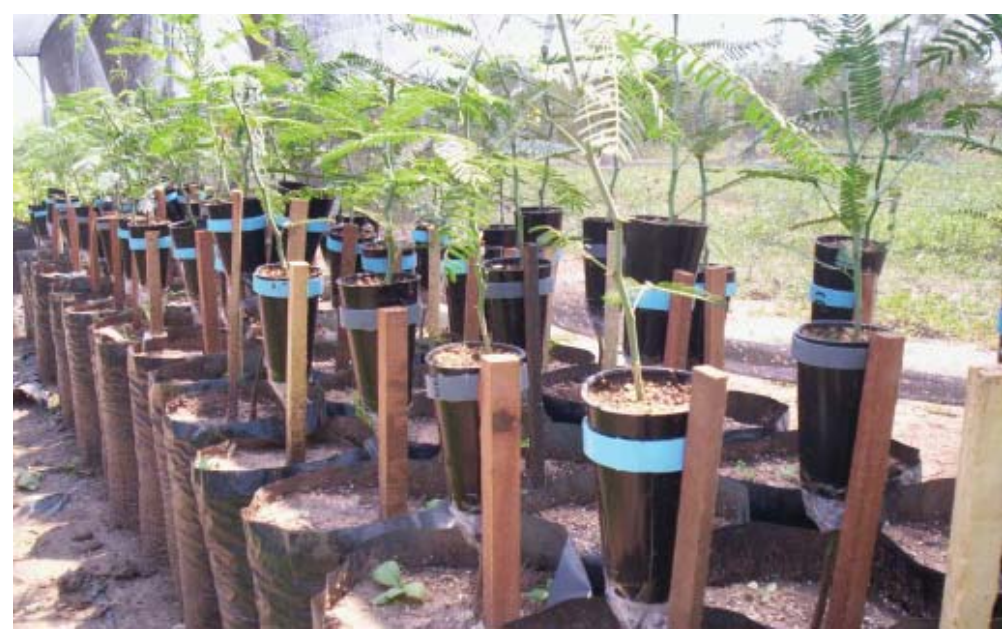

Figura 2. Acodos en montículos en plantas de Prosopis chilensis de 8 meses de edad. Figure 2. Mound layering on Prosopis chilensis 8-month-old plants.

Tabla 2. Tratamientos de acodo en montículo realizados con diferentes tipos de sustratos. Table 2. Mound layering treatments using different substrates.

\begin{tabular}{|l|c|c|c|c|}
\hline & \multicolumn{3}{|c|}{ Tipo de sustrato } & \\
\hline Tipo de acodo & arena & turba & arena + turba & Total \\
\hline Sin alambre & 24 & 24 & 24 & 72 \\
\hline Con alambre & 24 & 24 & 24 & 72 \\
\hline
\end{tabular}


se usó, respectivamente, el Test Exacto de Fisher y análisis de la varianza donde la diferencia de medias se determinó con el test LSD Fisher al $5 \%$ mediante el programa Infostat (Di Rienzo et al. 2016).

\section{RESULTADOS Y DISCUSIÓN}

\section{Acodo aéreo}

Determinación del efecto de la hormona enraizante ácido indolbutírico (AIB) y evaluación de la incidencia de la época de realización. En la Tabla 3 se pueden observar los resultados obtenidos de los tratamientos realizados.

Se comprobó que este método de clonación fue igualmente eficaz tanto en primavera como en verano, con o sin aplicación de AIB. En primavera se registró con AIB un promedio de $42,5 \%$ de acodos enraizados mientras que sin AIB fue del $30 \%$ (p $=0,257)$. En el ensayo de verano tampoco hubo una diferencia en el porcentaje de acodos enraizados con respecto al uso de esta hormona $(\mathrm{p}=0,46)$. Si bien la aplicación de AIB en primavera generó un 20 \% más de acodos enraizados que en verano, esta diferencia no fue significativa $(\mathrm{p}=0,062)$. Del mismo modo, Hagel y Palzkill (1989) usaron AIB a una dosis de 5.000 ppm (0,5 $\%)$ en primavera y en verano para acodar árboles de $P$. chilensis y no observaron diferencias entre ambas épocas. Vega et al. (1999) acodaron árboles de P. alba de 5 años de edad y encontraron que la mejor época fue en primavera y no en otoño, en el cual lograron obtener hasta un $85 \%$ de acodos enraizados. Es decir, la planta tiene que estar en pleno crecimiento activo para que el método dé resultados positivos. En este trabajo, el porcentaje de acodos enraizados sin AIB fue idéntico en ambas épocas (30 \%), similar a lo observado por Abdou et al. (2015) en ramas acodadas de árboles de $P$. africana sin el uso de ninguna hormona (28 \%).

En cuanto a la rizogénesis, esta fue similar $(p>0,05)$ en todos los tratamientos, sin importar la época de realización y el agregado de AIB. En este trabajo se obtuvieron en total 50 acodos enraizados de P. chilensis de 3 años de edad y el número promedio de raíces primarias fue de 14,5 entre los cuatro

Tabla 3. Resultados de los acodos aéreos en Prosopis chilensis realizados en dos épocas diferentes, con y sin aplicación de AIB, luego de 60 días de iniciado el tratamiento.

Table 3. Results of air layering in Prosopis chilensis carried out in two different seasons, with and without application of AIB, 60 days after treatment.

\begin{tabular}{|c|c|c|c|c|}
\hline $\begin{array}{c}\text { Época de } \\
\text { realización }\end{array}$ & $\begin{array}{c}\text { AIB } \\
(\%)\end{array}$ & $\begin{array}{c}\mathrm{N}^{\circ} \text { de acodos } \\
\text { enraizados }\end{array}$ & $\begin{array}{c}\text { Enraizamiento } \\
(\%)\end{array}$ & $\begin{array}{c}\mathrm{N}^{\circ} \text { promedio de } \\
\text { raíces primarias } \pm \mathrm{DS}\end{array}$ \\
\hline \multirow{2}{*}{ Primavera } & 1 & 17 & $42,5 \mathrm{a}$ & $16,0 \pm 9,6 \mathrm{a}$ \\
\cline { 2 - 5 } & 0 & 12 & $30,0 \mathrm{a}$ & $12,5 \pm 8,7 \mathrm{a}$ \\
\hline \multirow{2}{*}{ Verano } & 1 & 9 & $22,5 \mathrm{a}$ & $13,0 \pm 7,3 \mathrm{a}$ \\
\cline { 2 - 5 } & 0 & 12 & $30,0 \mathrm{a}$ & $16,5 \pm 10,8 \mathrm{a}$ \\
\hline Total & & 50 & & $14,5 \pm 9,1$ \\
\hline
\end{tabular}

Medias con una letra común no son estadísticamente diferentes diferentes $(p>0,05)$. DS = desvío estándar. 
tratamientos. En México lograron propagar por acodamiento árboles de 5 años de edad de Prosopis laevigata y observaron un promedio de 1156,2 raíces por acodo aéreo, mientras que en árboles adultos de 15 años de edad el número promedio de raíces por acodo fue 533, en ambos casos con el uso de la hormona AIB (Ramírez-Malagón et al. 2014). En este caso, los autores envolvieron el anillado con un papel embebido en la hormona y tal vez esa forma de aplicación fue lo que generó una gran cantidad de raíces adventicias, por ser más eficaz y con efectos duraderos, independientemente de la edad de los árboles.

Existen muchas variables a tener en cuenta al momento de ajustar este método de propagación. Además, se sabe que la capacidad de enraizamiento está influenciada por el genotipo de la planta madre (Vega et al. 2002a). Goel y Behl (1996) estudiaron la propagación vegetativa en siete especies de Prosopis de 3 años de edad y encontraron que $P$. pubescens Benth. tuvo la mejor capacidad de enraizamiento en los acodos que las demás especies. Otros autores observaron que en $P$. chilensis de 12 años de edad, los acodos enraizaron con una diferencia de hasta 3 semanas entre distintos árboles (Hagen y Palzkill 1989). Es decir, en Prosopis existiría una variación en la capacidad de enraizamiento debido a factores genéticos intra e interespecíficos. Es por ello que, probablemente, la gran variación en el número de raíces observado en este trabajo (DS=9,1) esté relacionado con la cantidad de plantas utilizadas. Además, otra variable que no se tuvo en cuenta fue la ubicación de la rama en la planta madre (altura con respecto al suelo), la posición del acodo en la rama elegida (basal o intermedia) y el diámetro de las ramas, aspectos que fueron estudiados por Abdou et al. (2015).

Se observó que en la época de verano los acodos enraizados que fueron tratados con AIB tuvieron, contrariamente a lo esperado, un sistema radicular adventicio menos desarrollado que los acodos no tratados (datos no medidos) (Figura 3).

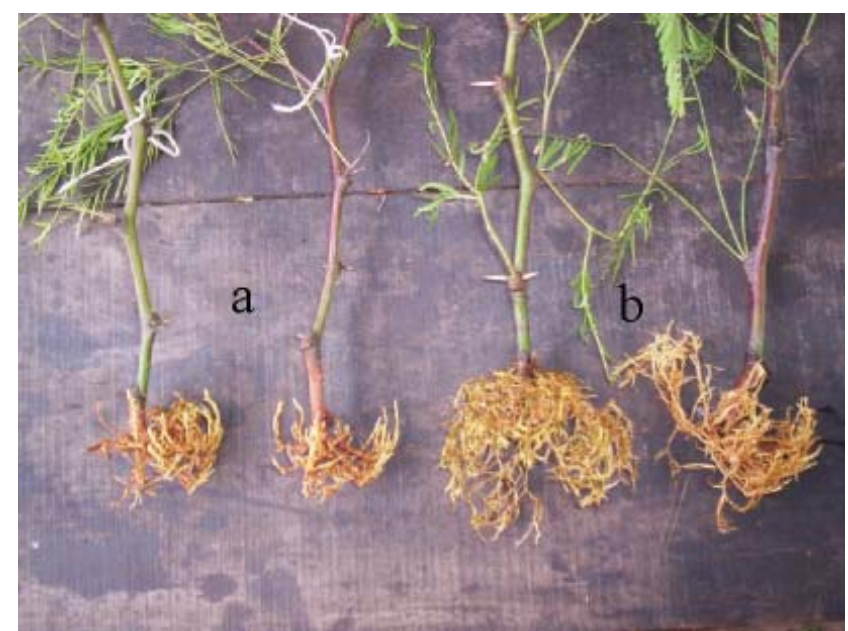

Figura 3. Acodos aéreos enraizados. a) dos ramas tratadas con AIB 1\%, b) dos ramas sin AIB. Figure 3. Rooting in air layering. a) two branches treated with AIB 1\%, b) two branches without AIB. 


\section{G. Tarnowski}

En general, los acodos tratados con AIB tienen un mayor porcentaje de enraizamiento y mejor calidad del sistema radicular que los no tratados (Hagen y Palzkill 1989). Es probable que la única dosis de AIB utilizada en este trabajo ( $1 \%$ ) tuviera un efecto más bien perjudicial que benéfico. Ramírez-Malagón et al. (2014) mencionaron que una elevada concentración hormonal mató la mayoría de los acodos realizados en ramas juveniles por eso debieron reducir las dosis. Según Hartmann y Kester (1987) la respuesta al enraizamiento no siempre es universal después del tratamiento con auxinas, es por ello que se piensa que ciertos cofactores estarían actuando de manera antagónica limitando el enraizado.

El empleo de la propagación agámica mediante acodo aéreo en especies de Prosopis es factible de realizarlo teniendo en cuenta que debería ajustarse en cada caso particular a los fines de optimizar los resultados.

\section{Acodo en montículo}

Determinación del efecto del estrangulado de los brotes y evaluación de la influencia del tipo de sustrato. El acodamiento genera una limitación del flujo de fotoasimilados, fitoreguladores, metabolitos y otros compuestos que favorecen la aparición de raíces adventicias en la posición del tallo donde existe una interrupción de los tejidos conductivos. En este ensayo la presencia del alambre indujo una diferencia significativa $(p<0,05)$ en la rizogénesis con respecto a las plantas testigos sin anillar.

En la Tabla 4 se pueden observar los resultados de los tratamientos realizados en este ensayo.

El porcentaje de acodos enraizados producidos en los sustratos A (75 \%) y AT (67 $\%)$ no fueron diferentes entre ellos, pero fueron significativamente superiores al sustrato T (42 \%) (p=0,02). Si bien en el sustrato $\mathrm{T}$ se registró el menor porcentaje de acodos enraizados, el número promedio

Tabla 4. Resultados de acodos en montículo en Prosopis chilensis realizados en plantas de 8 meses de edad con diferentes tipos de sustratos, luego de 60 días.

Table 4. Results of mound layering in Prosopis chilensis performed on 8-month-old plants with different types of substrates, 60 days after treatment.

\begin{tabular}{|c|c|c|c|c|}
\hline \multirow{4}{*}{ Con alambre } & Sustratos & $\begin{array}{c}\mathrm{N}^{0} \text { de acodos } \\
\text { enraizados }\end{array}$ & $\begin{array}{c}\text { Enraizamiento } \\
(\%)\end{array}$ & $\begin{array}{c}\mathrm{N}^{\circ} \text { promedio de } \\
\text { raíces primarias } \pm \mathrm{DS}\end{array}$ \\
\cline { 2 - 5 } & Turba & 10 & $42 \mathrm{a}$ & $5,50 \pm 2,53 \mathrm{a}$ \\
\cline { 2 - 5 } & Arena & 18 & $75 \mathrm{~b}$ & $3,89 \pm 2,27 \mathrm{~b}$ \\
\hline \multirow{3}{*}{ Sin alambre } & Turba & 16 & $67 \mathrm{~b}$ & $3,38 \pm 1,58 \mathrm{~b}$ \\
\cline { 2 - 5 } & Arena & 6 & $0 \mathrm{~d}$ & \\
\cline { 2 - 5 } & Arena + Turba & 0 & $25 \mathrm{c}$ & $1,83 \pm 0,98 \mathrm{c}$ \\
\hline
\end{tabular}

Medias con una letra común no son estadísticamente diferentes $(p>0,05)$. DS = desvío estándar 
de raíces adventicias $(5,5)$ fue mayor que en los otros dos sustratos $(p=0,03)$. En la Figura 4 se pueden observar las características de las raíces generadas en ambos sustratos: en turba son más gruesas, cortas y tienden a ser más frágiles; en arena, por el contrario, son más delgadas, largas y flexibles (datos no medidos).

La turba es un sustrato artificial orgánico que tiene propiedades tales como baja densidad, elevada porosidad, alta capacidad de aireación y retención de agua, en cambio la arena se comporta como un sustrato diferente, es poco poroso y retiene poca humedad porque favorece el drenaje del agua (Argo 1998, Brückner 1997, Salto et al. 2016). Valenzuela (2013) indica que es esencial el conocimiento de las propiedades físicas, químicas y biológicas de los sustratos usados y la medición de sus parámetros como son la porosidad total, capacidad de retención de agua y aire, $\mathrm{pH}$ y conductividad eléctrica. Si bien en este trabajo no se realizaron estos análisis, sería interesante profundizar con estos estudios a fin de investigar otras mezclas diferentes de sustratos y ajustar mejor el protocolo de propagación. Evidentemente, la presencia de arena en el sustrato se comportaría como un medio eficaz para el desarrollo radicular, tal como se puede observar en los altos porcentajes de rizogénesis en los sustratos A y AT con alambre. Inclusive, en las plantas que no fueron anilladas con alambre, únicamente se observaron acodos enraizados en el sustrato arena. De las 24 plantas tratadas enraizaron seis (25\%), con 1 a 3 raíces por acodo. Goel y Behl (1995) observaron que la arena sola era el mejor sustrato para el enraizamiento de estacas de P. juliflora (Swartz).

No existen referencias bibliográficas con respecto al uso de esta técnica en Prosopis. Recientemente, Coles y du Toit (2019) reportaron la utilización de esta metodología para clonar varias plantas de Moringa oleífera y obtuvieron $50 \%$ de acodos enraizados.

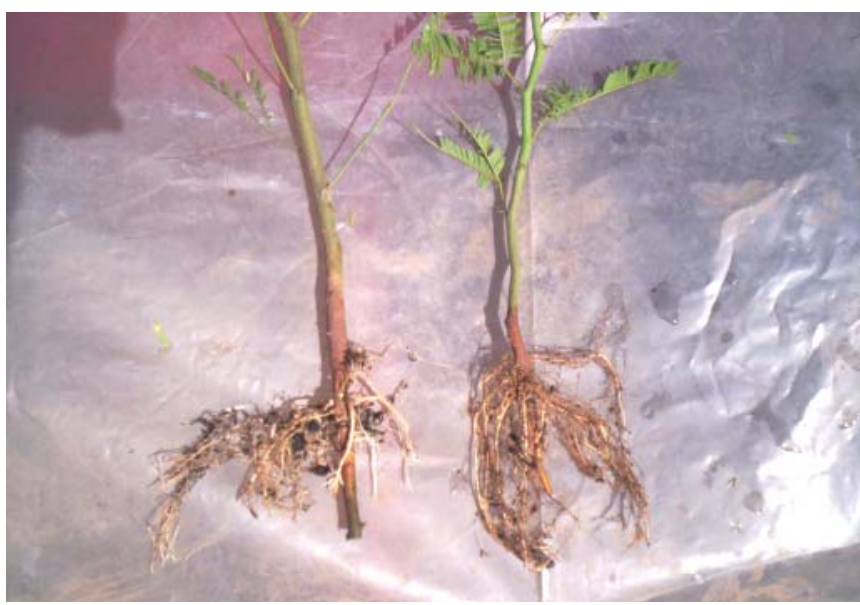

Figura 4. Características de las raíces formadas en sustrato turba, a la izquierda, y sustrato arena a la derecha.

Figure 4. Characteristics of the roots formed in peat substrate, on the left, and sand substrate on the right. 


\section{CONCLUSIÓN}

\section{Acodo aéreo}

No es necesaria la aplicación de AIB 1 $\%$ para obtener enraizamiento en los acodos aéreos, pero mejora ligeramente el porcentaje de prendimiento en primavera.

En cuanto a la época de realización de los acodos aéreos (primavera-verano) en ramas semileñosas del año, con diámetros de 0,5 a $1 \mathrm{~cm}$ y longitud mínima de $30 \mathrm{~cm}$, no inciden en el éxito del prendimiento y cantidad de raíces producidas.

\section{Acodo en montículo}

El estrangulado con alambre de $1 \mathrm{~mm}$ de grosor realizado en plantas de $P$. chilensis de 8 meses de edad, a una altura del tallo de $15 \mathrm{~cm}$, es efectivo para lograr el enraizamiento de los acodos en montículo.

La arena utilizada como sustrato mineral incrementa el porcentaje de enraizamiento de los acodos anillados pero produce menor cantidad de raíces que en el sustrato turba.

\section{Perspectivas de la técnica de acodamiento}

En este trabajo se probaron dos técnicas de acodamiento en algarrobo que pueden ser útiles para su aplicación en diferentes situaciones. Si el árbol es juvenil y presenta características de interés, por ejemplo, ausencia de espinas, se podría practicar el acodo aéreo como medio de propagación inicial y rejuvenecimiento. En cambio, si el árbol es adulto podrían presentarse dos alternativas: podar una rama gruesa y realizar acodos en los nuevos brotes al año siguiente $\mathrm{o}$, si se corta el árbol desde la base, se podrían hacer acodos en montículo a partir de los rebrotes. Se recomienda asimismo realizar estudios sobre los efectos de ciclófisis y topófisis en la capacidad de enraizamiento de los acodos realizados en plantas de $P$. chilensis, es decir, la ubicación de la rama en la planta madre (altura con respecto al suelo) y la posición del acodo en la rama elegida (basal o intermedia); además de otras características como ser el diámetro de las ramas y edad de la planta madre.

\section{AGRADECIMIENTOS}

Se agradece al Jefe del Grupo Forestal del INTA Yuto, el Ing. Ftal. Elvio del Castillo y al personal de apoyo por la ayuda y colaboración brindada en este trabajo, el cual fue realizado con financiamiento del Proyecto Regional Forestal del INTA: "Silvicultura Intensiva y Desarrollo Sustentable con Especies Valiosas en el Noroeste Argentino".

\section{BIBLIOGRAFÍA}

Abdou L, Karim S, Habou R, Mahamane A. 2015. Vegetative Propagation Trial of Prosopis africana

(Guill. et Perr.) Taub. by Air Layering under Sudano-Sahelian Climate in the South-Central Niger. Journal of Botany, 1-6.

Argo W. 1998. Root medium physical properties. Hort Technology 8(4):481-485.

Brückner U. 1997. Physical properties of different potting media and substrate mixtures - especially air - and water capacity. Proceeding on Growing Media and Plant Nutrition. Ed. R.U. Roeber Acta Hort. 450. ISHS. 263-270.

Burkart A. 1952. Las leguminosas argentinas, silvestres y cultivadas: Descripción sistemática de la familia, los géneros y las principales especies, de su distribución y 
utilidad en el país y en las regiones limítrofes. Acme Agency. Buenos Aires. 569 pp.

Carpineti L. 2005. Importancia de la Silvicultura Clonal. IDIA XXI Forestales. Año V. 8:153156.

Coles ZS, du Toit ES. 2019. Open air-layering of Moringa oleifera utilizing seedling plug containers. South African Journal of Botany. In press. https://doi.org/10.1016/j. sajb.2019.07.016

Del Castillo EM, Tarnowski CG. 2006. Cultivo Experimental De Prosopis chilensis (Molina) Stuntz. En El Pedemonte De Yungas. II Jornadas Forestales en Santiago del Estero: El árbol. Forestación y aprovechamiento integral del algarrobo. Online: https://fcf.unse.edu.ar/ eventos/2-jornadas-forestales/indx.htm

Di Rienzo JA, Casanoves F, Balzarini MG, Gonzalez L, Tablada M, Robledo CW. InfoStat versión 2016. Grupo InfoStat, FCA, Universidad Nacional de Córdoba, Argentina. URL http://www.infostat.com.ar

Goel VL, Behl HM. 1995. Propagation of Prosopis juliflora from rooted stem cuttings. International Tree Crops Journal 8:193-201.

Goel VL, Behl HM. 1996. Variation in thorn character within and among Prosopis species and vegetative multiplication of selected genotypes. Indian Journal of Forestry 19(2):132-136.

Hagen RH, Palzkill DA. 1989. Air-layering as a Method of Asexual Propagation of Mesquite. Turfgrass and Ornamentals Research Summary, 83-84.

Hartmann HT, Kester DE. 1987. Propagación de plantas. Principios y prácticas. Compañía Editorial Continental, S.A. de C.V., México. 760 pp.

Palacios R, Agullo MA, Mom MP, Torregrosa S, Picca P. 1988. Especies del Género. Taxonomía. Prosopis en Argentina, Documento Preliminar Elaborado Para el Primer Taller Internacional Sobre Recurso Genético y Con- servación de Germoplasma en Prosopis. FAO, FCAUNC y FCEy N-UBA, Córdoba. p. 1596.

Ramírez-Malagón R, Delgado-Bernal E, Borodanenko A, Pérez-Moreno L, Barrera-Guerra JL, Gordon Núñez-Palenius HY, Ochoa-Alejo N. 2014. Air Layering and Tiny-Air Layering Techniques for Mesquite Prosopis laevigata (H. B. ex Willd.) Johnst. M. C.] Tree Propagation. Arid Land Research and Management. 28:118-128.

Tapia AM, Clérici SE, Iriarte A. 2005. La multiplicación y propagación del algarrobo. Revista Chile Forestal. 319:17-19.

Salto C, Harrand L, Oberschelp GPJ, Ewens M. 2016. Crecimiento de plantines de Prosopis alba en diferentes sustratos, contenedores y condiciones de vivero. Bosque 37(3):527-537.

Valenzuela O. 2013. Las 5 llaves del mundo de los sustratos para plantas. Folleto informativo. INTA EEA San Pedro, Buenos Aires.

Vega MV, Castillo De Meier G, Pérez V, Bovo OA. 1999. Obtención de plantas de Prosopis alba (Leguminosae) mediante acodos aéreos. Phyton 65:83-86.

Vega MV, Castillo De Meier G, Bovo OA. 2002a. Efecto del genotipo sobre la propagación del género Prosopis aplicando la técnica de macropropagación. Novenas Jornadas Técnicas Forestales. INTA-FCF-MEYRNRYT-Eldorado, Misiones, Argentina.

Vega MV, Castillo De Meier G, Russo De Bordoy A, Bovo OA. 2002b. Enraizamiento de acodos aéreos realizados en árboles adultos de Prosopis alba Griseb. Novenas Jornadas Técnicas Forestales. INTA-FCF-MEYRNRYT-Eldorado, Misiones, Argentina.

Verga A, Córdoba A, Montura M, López Lauenstein D, Melchiorre M, Joseau J, Carranza C, Ledesma M, Recalde D, Tomalino L, et al. 2005. El Proyecto Algarrobo del INTA. IDIA XXI. Año V. 8:201-206. 